AperTO - Archivio Istituzionale Open Access dell'Università di Torino

\title{
Well, Better and In-Between
}

\section{This is a pre print version of the following article:}

Original Citation:

\section{Availability:}

This version is available http://hdl.handle.net/2318/1722705

since 2020-01-13T12:58:13Z

Publisher:

Springer

Published version:

DOI:10.1007/978-3-030-30229-0_1

Terms of use:

Open Access

Anyone can freely access the full text of works made available as "Open Access". Works made available under a Creative Commons license can be used according to the terms and conditions of said license. Use of all other works requires consent of the right holder (author or publisher) if not exempted from copyright protection by the applicable law. 


\title{
Well, Better and In-Between
}

\author{
Raphaël Carroy and Yann Pequignot*
}

June 18, 2017

\begin{abstract}
Starting from well-quasi-orders (wQOs), we motivate step by step the introduction of the complicated notion of better-quasi-order (BQO). We then discuss the equivalence between the two main approaches to defining BQO and state several essential results of BQO theory.

After recalling the rôle played by the ideals of a WQO in its BQOness, we give a new presentation of known examples of WQOs which fail to be BQO. We also provide new forbidden pattern conditions ensuring that a quasi-order is a better quasi-order.
\end{abstract}

\section{Contents}

1 Well is not good enough 3

2 Super-sequences versus multi-sequences $\quad 6$

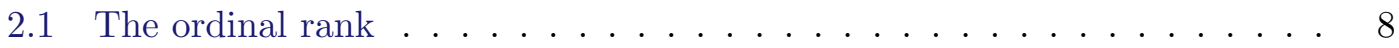

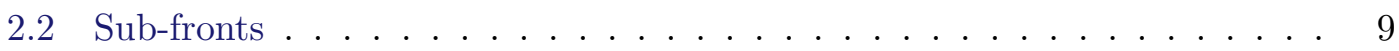

2.3 A Ramsey property for fronts: Nash-Williams's Theorem . . . . . . . . . 9

2.4 Continuous definition: multi-sequences . . . . . . . . . . . . . . 10

3 Well, here is better! 11

3.1 Two equivalent definitions . . . . . . . . . . . . . . . . . . . 11

3.2 First examples and finite stability . . . . . . . . . . . . . . . . 13

3.3 The real deal: infinite stability . . . . . . . . . . . . . . . . . 14

4 In-between: an intractable diversity of inconspicuous orders 18

5 Classes of bqos definable by forbidden pattern 22

5.1 Interval orders . . . . . . . . . . . . . . . . . 22

5.2 More classes of better-quasi-orders via forbidden patterns . . . . . . . . . 24

${ }^{*}$ The first author was supported by FWF Grants P28153 and P29999. The second author gratefully acknowledges the support of the Swiss National Science Foundation (SNF) through grant P2LAP2_164904. 
It is the variety of these applications, rather than any depth in the results obtained, that suggests that the theorems may be interesting.

$$
\text { Graham Higman [Hig52] }
$$

While studying a generalization of the partial order of divisibility on the natural numbers for an abstract algebra, Higman [Hig52] identified the following desirable property for a quasi-order (qo). A qo has the finite basis property if every upwards closed subset is the upward closure of a finite subset. He notices that this property is equivalent to that defining a well-quasi-order (WQO): being well-founded and having no infinite antichains. Higman proves the following essential fact: in order to be WQO it suffices to be generated by means of finitary operations from a WQO. He then proceeds to apply his theorem to solve a problem posed by Erdős, to provide a new proof of a theorem on power-series ring and also to the study of fully invariant subgroups of a free group. These were only the first instances of a long series of applications of this result that became known as Higman's Theorem. Pouzet [Pou85] later commented on the possibilities and the limitations of that fruitful approach:

In order to show that a certain class of posets (finite or infinite) is WQO, one tries first to see if the class can be constructed from some simpler class by means of some operations. If these operations are finitary, then it is possible that Higman's theorem can be applied. However, for infinite posets, these operations may very well also be infinitary and then there is no possibility of applying Higman's theorem since the obvious generalization of this is false for infinitary operations.

Pouzet here refers to the fact that well-founded quasi-orders, as well as wQOs, lack closure under certain infinitary operations as first proven by Rado [Rad54]. This is explained in detail in Section 1 where we point out how it opens the way to the definition by Nash-Williams [NW65] of the concept of better-quasi-order (BQO): a stronger property than WQO which allows for an infinitary analogue to Higman's Theorem. We first provide a gentle introduction to the original definition of Nash-William, before presenting the more concise definition introduced by Simpson [Sim85]. We use some new terminology with the hope that it makes it easier for the unacquainted reader to appreciate the respective advantages of these two complementary approaches to defining BQO.

To offer a few more words of introduction about this intriguing concept, we briefly comment on the emblematic case of the quasi-order $\operatorname{LIN}_{\aleph_{0}}$ of countable linear orders, equipped with the relation of embeddability. Fraïssé [Fra48] conjectured that $\operatorname{LIN}_{\aleph_{0}}$ was well-founded, but the statement that became known as Fraïssé's Conjecture (FRA) is that LIN $_{\aleph_{0}}$ is WQO; it follows from the famous theorem of Laver [Lav71] that LIN $_{\aleph_{0}}$ is in fact BQO. The reason for the use of the concept of BQO in Laver's proof of FRA was already alluded to in the above quote by Pouzet. While using Hausdorff's analysis of scattered linear orders and proceeding by induction is a very reasonable way to tackle FRA, the operations underlying this analysis are infinitary and this is a main obstacle 
when working with WQOs alone. This masterly use by Laver of the concept of BQO introduced by Nash-Williams inspired many other delightful results. But however successful this story is, it raises at least two questions.

Firstly, one may ask if the use of the concept of BQO in the proof of FRA is in a sense necessary. In the framework of Reverse Mathematics, one can formalize this question by asking for the exact proof-theoretic of FRA. The answer is still unknown despite many efforts, but important results have already been obtained [Mar05].

Secondly, one may ask if this strategy for proving wQOness always works. On the one hand, many other quasi-orders were proved to be BQO in the subsequent years attesting to the effectiveness of this concept (see Section 3.3). On the other hand, there does exist a large range of examples of WQO that are not BQO (see Section 4). Nevertheless, these examples appear to have a somehow artificial flavor since as Kruskal [Kru72, p.302] observed in his very nice historical introduction to WQO: "all 'naturally occurring' WQO sets which are known are BQO" 1 .

In a quest towards a deeper understanding of the discrepancy between WQO and BQO, we mention a result of ours on the role played by the ideals of a WQO in it being BQO (see Theorem 3.23). We show the relevance of this rather singular theorem by giving two applications of it. First, we use it in Section 4 to give a new presentation of examples of WQOs that, while failing to be BQOs, still enjoy stronger and stronger properties. Finally, we use this theorem to give in Section 5 some new conditions under which the two notions of WQO and BQO coincide.

\section{Well is not good enough}

In the sequel, $\left(Q, \leq_{Q}\right)$ always stands for a quasi-order, qo for short, i.e. a reflexive and transitive relation $\leq_{Q}$ on a non-empty set $Q$. . A sequence $\left(q_{n}\right)_{n \in \omega}$ in $Q$ is bad if and only if for all integers $m$ and $n$ such that $m<n$ we have $q_{m} \Varangle_{Q} q_{n}$.

The strict quasi-order associated to $\leq_{Q}$ is defined by $p<_{Q} q$ if and only if $p \leq_{Q} q$ and $q \Varangle_{Q} p$. We say that $Q$ is well-founded if there is no infinite descending chain in $Q$, i.e. no sequence $\left(q_{n}\right)_{n}$ such that $q_{n+1}<_{Q} q_{n}$ for every $n$. An antichain in $Q$ is a subset $A$ of $Q$ consisting of pairwise $\leq_{Q}$-incomparable elements, i.e. $p \neq q$ implies $p \not_{Q} q$ for every $p, q \in A$.

A subset $D$ of $Q$ is a called a downset, if $q \in D$ and $p \leq_{Q} q$ implies $p \in D$. For any $S \subseteq Q$, we write $\downarrow S$ for the downset generated by $S$ in $Q$, i.e. the set $\{q \in Q \mid \exists p \in$ $\left.S q \leq_{Q} p\right\}$. We also write $\downarrow p$ for $\downarrow\{p\}$. Finally we denote by $\mathcal{D}(Q)$ the po of downsets of $Q$ under inclusion.

We start by proving the equivalence between three of the main characterizations of a WQO.

Proposition 1.1. A quasi-order $\left(Q, \leq_{Q}\right)$ is a WQO if and only if one of the following equivalent conditions is fulfilled:

${ }^{1}$ The minor relations on finite graphs, proved to be WQO by Robertson and Seymour[RS04], is to our knowledge the only naturally occurring WQO which is not yet known to be BQO. 
1. there is no bad sequence in $\left(Q, \leq_{Q}\right)$,

2. $\left(Q, \leq_{Q}\right)$ is well-founded and contains no infinite antichain,

3. $(\mathcal{D}(Q), \subseteq)$ is well-founded.

Proof. Item $2 \leftrightarrow$ Item 1 Notice that an infinite descending chain and a countably infinite antichain are both sepcial cases of a bad sequence. Conversely if $\left(q_{n}\right)_{n}$ is a bad sequence in $Q$, then using Ramsey's theorem we obtain either a infinite descending chain or an infinite antichain.

Item $\mathbf{1} \rightarrow$ Item 3 By contraposition, suppose that $\left(D_{n}\right)_{n \in \omega}$ is an infinite descending chain inside $(\mathcal{D}(Q), \subseteq)$. Then for each $n \in \omega$ we can pick some $q_{n} \in D_{n} \backslash D_{n+1}$. Then $n \mapsto q_{n}$ is a bad sequence in $Q$. To see this, suppose towards a contradiction that for $m<n$ we have $q_{m} \leq q_{n}$. As $q_{n} \in D_{n}$ and $D_{n}$ is a downset, we have $q_{m} \in D_{n}$. But since $D_{n} \subset D_{m+1}$, we have $q_{m} \in D_{m+1}$, a contradiction with the choice of $q_{m}$.

Item $3 \rightarrow$ Item 1 By contraposition, suppose that $\left(q_{n}\right)_{n \in \omega}$ is a bad sequence in $Q$. Set $D_{n}=\downarrow\left\{q_{k} \mid n \leq k\right\}$, then $\left(X_{n}\right)_{n}$ is a descending chain in $\mathcal{D}(Q)$. Indeed for every $n$ we clearly have $D_{n+1} \subseteq D_{n+1}$ and since the sequence is bad, $k \geq n+1$ implies $q_{n} \not \leq q_{k}$ and so $q_{n} \in D_{n}$ while $q_{n} \notin X_{n+1}$.

After Proposition 1.1, it is natural to ask if being well-founded and being wQO is actually equivalent for the partial order of downsets of any quasi-order. The answer is negative and the first example of a WQO with an antichain of downsets was identified by Richard Rado.

Example 1.2 ([Rad54]). Rado's partial order $\mathfrak{R}$ is the set $[\omega]^{2}$ of pairs of natural numbers, partially ordered by (cf. Fig. 1):

$$
\{m, n\} \leq_{\mathfrak{R}}\left\{m^{\prime}, n^{\prime}\right\} \longleftrightarrow\left\{\begin{array}{l}
m=m^{\prime} \text { and } n \leq n^{\prime} \text {, or } \\
n<m^{\prime}
\end{array}\right.
$$

where by convention a pair $\{m, n\}$ of natural numbers is always assumed to be written in increasing order $(m<n)$.

The po $\mathfrak{R}$ is WQO. To see this, consider any map $f: \omega \rightarrow[\omega]^{2}$ and let $f(n)=$ $\left\{f_{0}(n), f_{1}(n)\right\}$ for all $n \in \omega$. Now if $f_{0}$ is unbounded, then there exists $n>0$ with $f_{1}(0)<f_{0}(n)$ and so $f(0) \leq_{\Re} f(n)$ by the second clause. So $f$ is good in this case. Next if $f_{0}$ is bounded, then by going to a subsequence we can assume that $f_{0}$ is constantly equal to some $k$. But then the restriction of $\mathfrak{R}$ to the pairs $\left\{k, n_{1}\right\}$ is simply $\omega$ which is WQO, so $f$ must be good in this case too.

However the map $n \mapsto D_{n}=\downarrow\{\{n, l\} \mid n<l\}$ is a bad sequence (in fact an infinite antichain) inside $\mathcal{D}(\mathfrak{R})$. Indeed whenever $m<n$ we have $\{m, n\} \in D_{m}$ while $\{m, n\} \notin$ $D_{n}$, and so $D_{m} \nsubseteq D_{n}$.

Suppose we want to make sure that $\mathcal{D}(Q)$ is wQO. What condition on $Q$ could ensure this? In other words, what phenomenon are we to exclude inside $Q$ in order to rule out 


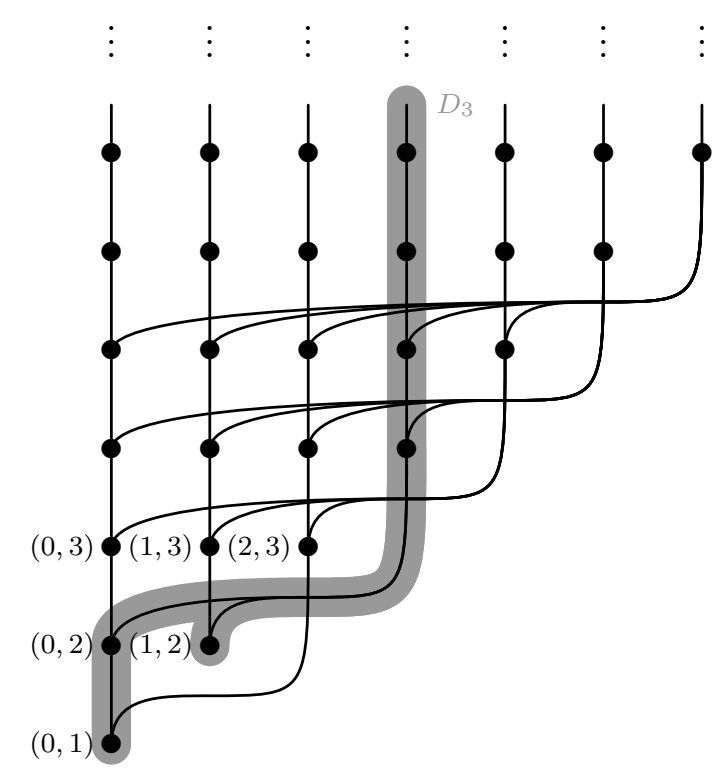

Figure 1: Rado's poset $\Re$.

the existence of antichains inside $\mathcal{D}(Q)$ ? Forbidding bad sequences in $Q$ is certainly not enough, as shown by the existence of Rado's example. But here is what we can do.

Suppose that $\left(P_{n}\right)_{n \in \omega}$ is a bad sequence in $\mathcal{D}(Q)$. Fix some $m \in \omega$. Then whenever $m<n$ we have $P_{m} \nsubseteq P_{n}$ and we can choose a witness $q \in P_{m} \backslash P_{n}$. In general, no single $q \in P_{m}$ can witness that $P_{m} \nsubseteq P_{n}$ for all $n>m$, so we have to pick a whole sequence $f_{m}: \omega / m \rightarrow Q, n \mapsto q_{m}^{n}$ of witnesses ${ }^{2}:$

$$
q_{m}^{n} \in P_{m} \quad \text { and } \quad q_{m}^{n} \notin P_{n}, \quad n \in \omega / m .
$$

In this way we get a sequence $f_{0}, f_{1}, \ldots$ of sequences which is advantageously viewed as single map from $[\omega]^{2}$ :

$$
\begin{aligned}
& f:[\omega]^{2} \longrightarrow Q \\
& \{m, n\} \longmapsto f_{m}(n)=q_{m}^{n} .
\end{aligned}
$$

By our choices this sequence of sequences satisfies the following condition:

$$
\forall m, n, l \in \omega \quad m<n<l \rightarrow q_{m}^{n} \not \leq q_{n}^{l} .
$$

To see this, suppose towards a contradiction that for $m<n<l$ we have $q_{m}^{n} \leq q_{n}^{l}$. Since $q_{n}^{l} \in P_{n}$ which is a downset, we would have $q_{m}^{n} \in P_{n}$, but we chose $q_{m}^{n}$ such that $q_{m}^{n} \notin P_{n}$.

Let us say that a sequence of sequences $f:[\omega]^{2} \rightarrow Q$ is bad if for every $m, n, l \in \omega$, $m<n<l$ implies $f(\{m, n\}) \not \leq f(\{n, l\})$. We have found the desired condition.

\footnotetext{
${ }^{2}$ where $\omega / m$ denotes the set $\{n \in \omega \mid m<n\}$.
} 
Proposition 1.3. Let $Q$ be a qo. Then $\mathcal{D}(Q)$ is WQO if and only if there is no bad sequence of sequences in $Q$.

Proof. As we have seen in the preceding discussion, if $\mathcal{D}(Q)$ is not wQO then from a bad sequence in $\mathcal{D}(Q)$ we can define a bad sequence of sequences in $Q$.

Conversely, if $f:[\omega]^{2} \rightarrow Q$ is a bad sequence of sequences, then for each $m \in \omega$ we can consider the set $P_{m}=\{f(\{m, n\}) \mid n \in \omega / m\}$ consisting in the image of the $m^{\text {th }}$ sequence. Then the sequence $m \mapsto \downarrow P_{m}$ in $\mathcal{D}(Q)$ is a bad sequence. Indeed every time $m<n$ we have $f(\{m, n\}) \in P_{m}$ while $f(\{m, n\}) \notin \downarrow P_{n}$, since otherwise there would exist $l>n$ with $f(\{m, n\}) \leq f(\{n, l\})$, a contradiction with the fact that $f$ is a bad sequence of sequences.

Notice that in the case of Rado's partial order $\mathfrak{R}$, the identity map itself is a bad sequence of sequences witnessing that $\mathcal{D}(\mathfrak{R})$ is not WQO, since every time $m<n<l$ then $\{m, n\} \Varangle_{\mathfrak{R}}\{n, l\}$. This example is actually minimal in the following sense; if $Q$ is WQO but $\mathcal{D}(Q)$ is not WQO, then $\mathfrak{R}$ embeds into $Q$, as proved by Laver [Lav76].

For now, let us just say that a better-quasi-order is a quasi-order $Q$ such that $\mathcal{D}(Q)$ is well-founded, $\mathcal{D}(\mathcal{D}(Q))$ is well founded, $\mathcal{D}(\mathcal{D}(\mathcal{D}(Q)))$ is well-founded, so on and so forth into the transfinite. While this idea can be formalized, we can already see that it cannot serve as a convenient definition ${ }^{3}$.

In the next Section, we introduce the super-sequences and the multi-sequences which are two equivalent way of generalizing the idea of sequence of sequences into the transfinite. This allows us to define better-quasi-orders in Section 3.

\section{Super-sequences versus multi-sequences}

As the preceding section suggests, we are going to define a better-quasi-order as a quasiorder with no bad sequence, with no bad sequence of sequences, no bad sequence of sequences of sequences, so on and so forth, into the transfinite. In order to formalize this idea, we need a convenient notion of "index set" for a sequence of sequences of ... of sequences, in short a super-sequence. We first describe the original combinatorial approach of Nash-Williams, before presenting the more condensed topological definition due to Simpson.

Let us first fix some notations. We adopt the set-theoretic convention that $n \in n+1$ for all natural number $n$. A sequence is a map from an initial segment of $\omega$ to some non-empty set, a finite sequence $s$ has domain an integer $n$, also called the length of $s$ and denoted by $|s|$. When $i<|s|, s(i)$ stands for the $i$-th element of the sequence $s$. If $A$ is a non-empty set, $A^{<\omega}$ stands for the set of finite sequences in $A$ and $A^{\omega}$ stands for the set of infinite sequences in $A$.

We write $[A]^{<\infty}$ for the set of finite subsets of $A$ and $[A]^{\infty}$ for the set of infinite subsets of $A$. We identify any subset of the natural numbers with its increasing enumeration.

\footnotetext{
${ }^{3}$ The reader who remains unconvinced can try to prove that the partial order $(3,=)$ satisfies this property.
} 
In this way, we say that $t \subseteq \omega$ extends $s \subseteq \omega$, in symbol $s \sqsubseteq t$, exactly when this happens for the corresponding increasing enumerations.

Definition 2.1. A family $F \subseteq[\omega]^{<\infty}$ is a front on $X \in[\omega]^{\infty}$ if

1. either $F=\{\emptyset\}$, or $\bigcup F=X$,

2. for all $s, t \in F s \sqsubseteq t$ implies $s=t$,

3. (Density) for all $X^{\prime} \in[X]^{\infty}$ there is an $s \in F$ such that $s \sqsubset X^{\prime}$.

A super-sequence in a set $Q$ is a map from any front $F$ on $X \in[\omega]^{\infty}$ to $Q$.

Notice that, according to our definition, the trivial front $\{\emptyset\}$ is a front on $X$ for every $X \in[\omega]^{\infty}$. Except for this degenerate example, if a family $F \subseteq[X]^{<\infty}$ is a front on $X$, then necessarily $X$ is equal to $\bigcup F$, the set-theoretic union of the family $F$. For this reason we will sometimes say that $F$ is a front, without reference to any infinite subset $X$ of $\omega$. Moreover when $F$ is not trivial, we refer to the unique $X$ for which $F$ is a front on $X$, namely $\bigcup F$, as the base of $F$.

Example 2.2. For all natural number $n$ the set $[\omega]^{n+1}$ is a non-trivial front. The family $\mathcal{S}:=\left\{s \in[\omega]^{<\infty}:|s|=s(0)+1\right\}$ is also a front, it is traditionnally called the Schreier barrier.

A sequence of sequences is a super-sequence with domain $[\omega]^{2}$, a sequence of sequences of sequences is a super-sequence with domain $[\omega]^{3}$, and so on. A super-sequence with domain $\mathcal{S}$ is an example of a transfinite super-sequence.

A front can be profitably decomposed in a sequence of "simpler" fronts.

Fact 2.3. Let $F$ be a front on $X \in[\omega]^{\infty}$. For all $n \in X$ the set $F_{n}:=\left\{s \in[\omega / n]^{<\infty} \mid\right.$ $\{n\} \cup s \in F\}$ is a front on $X / n$ called the ray of $F$ at $n$. Moreover $F=\bigcup_{n \in X}\{\{n\} \cup s \mid$ $\left.s \in F_{n}\right\}$.

Proof. Let $n \in X$. For every $Y \in[X / n]^{\infty}$ there exists $s \in F$ with $s \sqsubset\{n\} \cup Y$. Since $F$ is non trivial, $s \neq \emptyset$ and so $n \in s$. Therefore $s^{\prime}=s \backslash\{n\} \in F_{n}$ with $s^{\prime} \sqsubset Y$, and $F_{n}$ satisfies Item 3. Now if $F_{n}$ is not trivial and $k \in X / n$, there is $s \in F_{n}$ with $s \sqsubset\{k\} \cup X / k$ and necessarily $k \in s \subseteq \bigcup F_{n}$. Hence $\bigcup F_{n}=X / n$, so Item 1 is met. To see Item 2 , let $s, t \in F_{n}$ with $s \sqsubseteq t$. Then for $s^{\prime}=\{n\} \cup s$ and $t^{\prime}=\{n\} \cup t$ we have $s^{\prime}, t^{\prime} \in F$ and $s^{\prime} \sqsubseteq t^{\prime}$, so $s^{\prime}=t^{\prime}$ and $s=t$, as desired. The last statement is obvious.

Generalizing Ramsey's theorem, Nash-Williams proved that fronts enjoy a fundamental property: any time we partition a front into finitely many pieces, at least one of the pieces contains a front. We are now going to introduce the necessary tools to prove this result: first, an ordinal rank on fronts that allows for inductive proofs, then a characterization of what a sub-front looks like. 


\subsection{The ordinal rank}

We define a rank on fronts by associating to every given front a well-founded tree. We first need some classical notations and definitions about trees.

Definitions 2.4. $\quad$ 1. A tree $T$ on a set $A$ is a subset of $A^{<\omega}$ that is closed under prefixes, i.e. $u \sqsubseteq v$ and $v \in T$ implies $u \in T$.

2. A tree $T$ on $A$ is called well-founded if $T$ has no infinite branch, i.e. if there is no infinite sequence $x \in A^{\omega}$ such that $\left.x\right|_{n} \in T$ holds for all $n \in \omega$. In other words, a tree $T$ is well-founded if $(T, \sqsupseteq)$ is a well-founded partial order.

3. When $T$ is a non-empty well-founded tree we can define a strictly decreasing function $\rho_{T}$ from $T$ to the ordinals by transfinite recursion:

$$
\rho_{T}(t)=\sup \left\{\rho_{T}(s)+1 \mid t \sqsubset s \in T\right\} \quad \text { for all } t \in T .
$$

It is easily shown to be equivalent to

$$
\rho_{T}(t)=\sup \left\{\rho_{T}\left(t^{\frown}(a)\right)+1 \mid a \in A \text { and } t^{\frown}(a) \in T\right\} \quad \text { for all } t \in T .
$$

The rank of the non-empty well-founded tree $T$ is the ordinal $\rho_{T}(\emptyset)$.

Through the identification of a set of natural numbers with its increasing enumeration we can consider the tree generated by a front. For any front $F$, we let $T(F)$ be the smallest tree on $\omega$ containing $F$, i.e.

$$
T(F)=\left\{s \in \omega^{<\omega} \mid \exists t \in F s \sqsubseteq t\right\} .
$$

Lemma 2.5. For every front $F$, the tree $T(F)$ is well-founded.

Proof. If $x$ is an infinite branch of $T(F)$, then $x$ enumerates an infinite subset $X$ of $\bigcup F$ such that for every $u \sqsubset X$ there exists $t \in F$ with $u \sqsubseteq t$. Since $F$ is a front there exists a (unique) $s \in F$ with $s \sqsubset X$. But for $n=\min X / s, u=s \cup\{n\}$ and there is $t \in F$ with $u \sqsubseteq t$. But then $F \ni s \sqsubset u \sqsubseteq t \in F$ contradicting Item 2 in the definition of a front.

Definition 2.6. Let $F$ be a front. The rank of $F$, denoted by $\operatorname{rk} F$, is the rank of the tree $T(F)$.

Example 2.7. Notice that the family $\{\emptyset\}$ is the only front of null rank, and for all positive integer $n$, the front $[\omega]^{n}$ has rank $n$. Moreover the Schreier barrier $\mathcal{S}$ has rank $\omega$.

We now observe that the rank of $F$ is closely related to the rank of its rays $F_{n}, n \in X$. Given $F$ a non trivial front on $X \in[\omega]^{\infty}$, notice that the tree $T\left(F_{n}\right)$ of the front $F_{n}$ is naturally isomorphic to the subset

$$
\{s \in T(F) \mid\{n\} \sqsubseteq s\}
$$

of $T(F)$. The rank of the front $F$ is therefore related to the ranks of its rays through the following formula:

$$
\operatorname{rk} F=\sup \left\{\operatorname{rk}\left(F_{n}\right)+1 \mid n \in X\right\} .
$$

In particular, $\operatorname{rk} F_{n}<\operatorname{rk} F$ for all $n \in X$.

This simple remark allows one to prove results on fronts by induction on the rank by applying the induction hypothesis to the rays, as it was first done in [PR82]. 


\subsection{Sub-fronts}

By analogy with classical sequences let us make the following definition.

Definition 2.8. A sub-super-sequence of a super-sequence $f: F \rightarrow E$ is a restriction $f\left\lceil_{G}: G \rightarrow E\right.$ to some front $G$ included in $F$.

The following important operation is quite useful when dealing with sub-fronts of a given front, i.e. sub-families of a front which are themselves fronts. For a family $F \subseteq \mathcal{P}(\omega)$ and some $X \in[\omega]^{\infty}$, we define the sub-family

$$
F \mid X:=\{s \in F \mid s \subseteq X\} .
$$

Proposition 2.9. Let $F$ be a front on $X$. Then a family $F^{\prime} \subseteq F$ is a front if and only if there exists $Y \in[X]^{\infty}$ such that $F \mid Y=F^{\prime}$.

Proof. The claim is obvious if $F$ is trivial so suppose $F$ is non-trivial.

$\rightarrow$ Let $F^{\prime} \subseteq F$ be a front on $Y$. Since $F^{\prime}$ is not trivial either, $Y=\bigcup F^{\prime} \subseteq \bigcup F=X$. Now if $s \in F^{\prime}$ then clearly $s \in F \mid Y$. Conversely if $s \in F \mid Y$ then there exists a unique $t \in F^{\prime}$ with $t \sqsubset s \cup Y / s$ and so either $s \sqsubseteq t$ or $t \sqsubseteq s$. Since $F$ is a front and $s, t \in F$, necessarily $s=t$ and so $s \in F^{\prime}$. Therefore $F^{\prime}=F \mid Y$.

$\leftarrow$ If $Y \in[X]^{\infty}$ then the family $F \mid Y$ is a front on $Y$. Clearly $F \mid Y$ satisfies Item 2. If $Z \in[Y]^{\infty}$ then since $Y \subseteq X$, then $Z \in[X]^{\infty}$ and so there exists $s \in F$ with $s \sqsubset Z$. But then $s \subseteq Z \subseteq Y$, so in fact $s \in F \mid Y$ and therefore $F \mid Y$ satisfies Item 3. For Item 1, notice that $\bigcup F \mid Y \subseteq Y$ by definition and that if $n \in Y$, then as we have already seen there exists $s \in F \mid Y$ with $s \sqsubset\{n\} \cup Y / n$, so $n \in s$ and $n \in \bigcup F \mid Y$.

Observe that the operation of restriction commutes with the taking of rays.

Fact 2.10. Let $F \subseteq \mathcal{P}(\omega)$ and $X \in[\omega]^{\infty}$. For every $n \in X$ we have

$$
F_{n} \mid X=(F \mid X)_{n}
$$

Notice also the following simple important fact. If $F^{\prime}$ is a sub-front of a front $F$, then the tree $T\left(F^{\prime}\right)$ is included in the tree $T(F)$ and so $\operatorname{rk} F^{\prime} \leq \operatorname{rk} F$.

\subsection{A Ramsey property for fronts: Nash-Williams's Theorem}

We now prove this theorem to give a simple example of a proof by induction on the rank of a front, an extremely fruitful technique.

Theorem 2.11 (Nash-Williams). Let $F$ be a front. For any subset $S$ of $F$ there exists a front $F^{\prime} \subseteq F$ such that either $F^{\prime} \subseteq S$ or $F^{\prime} \cap S=\emptyset$.

Proof. The claim is obvious for the trivial front whose only subsets are the empty set and the whole trivial front. So suppose that the claim holds for every front of rank 
smaller than $\alpha$. Let $F$ be a front on $X$ with $\operatorname{rk} F=\alpha$ and $S \subseteq F$. For every $n \in X$ let $S_{n}$ be the subset of the ray $F_{n}$ given by $S_{n}=\left\{s \in F_{n} \mid\{n\} \cup s \in S\right\}$.

Set $X_{-1}=X$ and $n_{0}=\min X_{-1}$. Since rk $F_{n_{0}}<\alpha$ there exists by induction hypothesis some $X_{0} \in\left[X_{-1} / n_{0}\right]^{\infty}$ such that

$$
\text { either } F_{n_{0}} \mid X_{0} \subseteq S_{n_{0}}, \quad \text { or } F_{n_{0}} \mid X_{0} \cap S_{n_{0}}=\emptyset .
$$

Set $n_{1}=\min X_{0}$. Now applying the induction hypothesis to $F_{n_{1}} \mid\left(X_{0} / n_{0}\right)$ and $S_{n_{1}}$ we get an $X_{1} \in\left[X_{0} / n_{0}\right]^{\infty}$ such that either $F_{n_{1}} \mid X_{1} \subseteq S_{n_{1}}$, or $F_{n_{1}} \mid X_{1} \cap S_{n_{1}}=\emptyset$. Continuing in this fashion, we obtain a sequence $X_{k}$ together with $n_{k}=\min X_{k-1}$ such that for all $k$ we have $X_{k} \in\left[X_{k-1} / n_{k}\right]^{\infty}$ and

$$
\text { either } F_{n_{k}} \mid X_{k} \subseteq S_{n_{k}}, \quad \text { or } F_{n_{k}} \mid X_{k} \cap S_{n_{k}}=\emptyset .
$$

Now there exists $Y \in[\omega]^{\infty}$ such that either $F_{n_{k}} \mid X_{k} \subseteq S_{n_{k}}$ for all $k \in Y$, or $F_{n_{k}} \mid X_{k} \cap S_{n_{k}}=$ $\emptyset$ for all $k \in Y$. Let $X=\left\{n_{k} \mid k \in Y\right\}$ then $F \mid X$ is as desired. Indeed for all $s \in F \mid X$ we have min $s=n_{k}$ for some $k \in Y$ and $s \backslash\left\{n_{k}\right\} \in F_{n_{k}} \mid X_{k}$. Hence by the choice of $Y$, either $s \backslash\{\min s\} \in S_{\min s}$ for all $s \in F \mid X$, or $s \backslash\{\min s\} \notin S_{\min s}$ for all $s \in F \mid X$. Therefore either $F \mid X \subseteq S$ or $F \mid X \cap S=\emptyset$.

Nash-Williams' Theorem 2.11 is easily seen to be equivalent to the following statement.

Theorem 2.12. Let $E$ be a finite set. Then every super-sequence $f: F \rightarrow E$ admits a constant sub-super-sequence.

The above result obviously does not hold in general for an infinite set $E$ (consider for example any injective super-sequence). However [PR82] proved an interesting theorem in this context. In a different direction the authors also obtained in [CP14] that when $E$ is a compact metric space, then every super-sequence $f: F \rightarrow E$ admits a sub-supersequence which is a so-called Cauchy super-sequence.

\subsection{Continuous definition: multi-sequences}

We now present another fruitful approach to the definition of better-quasi-orders, initiated by [Sim85], and we relate it to super-sequences.

Let $E$ be any set, and $f: F \rightarrow E$ be a super-sequence with $F$ a front on $X$. For every $Y \in[X]^{\infty}$ there exists a unique $s \in F$ with $s \sqsubset Y$. We can therefore define a map $f^{\uparrow}:[X]^{\infty} \rightarrow E$ defined by $f^{\uparrow}(Y)=f(s)$ where $s$ is the unique member of $F$ with $s \sqsubset Y$.

Definition 2.13. A multi-sequence in some set $E$ is a map $h:[X]^{\infty} \rightarrow E$ for some $X \in[\omega]^{\infty}$. A sub-multi-sequence of $h:[X]^{\infty} \rightarrow E$ is a restriction of $h$ to $[Y]^{\infty}$ for some $Y \in[X]^{\infty}$.

For every $X \in[\omega]^{\infty}$ we endow $[X]^{\infty}$ with the topology induced by the Cantor space, identifying once again subsets of the natural numbers with their characteristic functions. As a topological space $[X]^{\infty}$ is homeomorphic to the Baire space $\omega^{\omega}$. This homeomorphism is conveniently realized via the embedding of $[X]^{\infty}$ into $\omega^{\omega}$ which maps each 
$Y \in[X]^{\infty}$ to its injective and increasing enumeration $e_{Y}: \omega \rightarrow Y$. We henceforth identify the space $[X]^{\infty}$ with the closed subset of $\omega^{\omega}$ of injective and increasing sequences in $X$. We thus obtain a countable basis of clopen sets for $[X]^{\infty}$ :

$$
M_{s}=N_{s} \cap[X]^{\infty}=\left\{Y \in[X]^{\infty} \mid s \sqsubset Y\right\}, \quad \text { for } s \in[X]^{<\infty} .
$$

Definition 2.14. A multi-sequence $h:[X]^{\infty} \rightarrow E$ is locally constant if for all $Y \in[X]^{\infty}$ there exists $s \in[X]^{<\infty}$ such that $Y \in M_{s}$ and $h$ is constant on $M_{s}$, i.e. for every $Y \in[X]^{\infty}$ there exists $s \sqsubset Y$ such that for every $Z \in[X]^{\infty}, s \sqsubset Z$ implies $h(Z)=h(Y)$.

Clearly for every super-sequence $f: F \rightarrow E$ where $F$ is a front on $X$ the map $f^{\uparrow}:[X]^{\infty} \rightarrow E$ is locally constant.

Conversely for any locally constant multi-sequence $h:[X]^{\infty} \rightarrow E$, let

$$
S^{h}=\left\{s \in[X]^{<\infty} \mid h \text { is constant on } M_{s}\right\} .
$$

Lemma 2.15. The set $F^{h}$ of $\sqsubseteq$-minimal elements of $S^{h}$ is a front on $X$.

Proof. By $\sqsubseteq$-minimality if $s, t \in F^{h}$ and $s \sqsubseteq t$, then $s=t$. For every $Y \in[X]^{\infty}$, since $h$ is locally constant there exists $s \sqsubset Y$ such that $h$ is constant on $M_{s}$. Hence there exists $t \in F^{h}$ with $t \sqsubseteq s$, and so $t \sqsubset Y$ too. To see that either $F^{h}$ is trivial or $\bigcup F^{h}=X$, notice that $h$ is constant if and only if $F^{h}$ is the trivial front if and only if $\emptyset \in F_{h}$. So if $F^{h}$ is not trivial, then for every $n \in X$ there exists $s \in F^{h}$ with $s \sqsubset\{n\} \cup X / n$ and since $s \neq \emptyset$, we get $n \in s$ and $n \in \bigcup F^{h}$.

We can therefore associate to every locally constant multi-sequence $h:[X]^{\infty} \rightarrow E$ a super-sequence $h^{\downarrow}: F^{h} \rightarrow E$ by letting, in the obvious way, $h^{\downarrow}(s)$ be equal to the unique value taken by $h$ on $M_{s}$ for every $s \in F^{h}$.

Corollary 2.16. Let $E$ be a finite set. Then every multi-sequence $f:[X]^{\infty} \rightarrow E$ admits a constant sub-multi-sequence.

\section{Well, here is better!}

As promised, we now give the two main definitions of BQO available in the literature. Proceeding in unchronological order, we start by the one due to Simpson which makes use of multi-sequences before stating the original one due to Nash-Williams based on supersequences. In both definitions, we only miss one last ingredient: a suitable generalization of the usual order on the natural numbers.

\subsection{Two equivalent definitions}

For every $N \in[\omega]^{\infty}$ we call the shift of $N$, denoted by ${ }_{*} N$, the set $N \backslash\{\min N\}$. Notice that $N \mapsto{ }_{*} N$ is a continuous map from $[\omega]^{\infty}$ to itself.

Definition 3.1. Let $Q$ be a qo and $h:[X]^{\infty} \rightarrow Q$ a multi-sequence. 
1. We say that $h$ is bad if $h(N) \not \leq h\left({ }_{*} N\right)$ for every $N \in[X]^{\infty}$,

2. We say that $h$ is good if there exists $N \in[X]^{\infty}$ with $h(N) \leq h\left({ }_{*} N\right)$,

At last, we present the deep definition due to Nash-Williams here in its modern "Simpsonian" reformulation.

Definition 3.2. A quasi-order $Q$ is a better-quasi-order (BQO) if there is no bad locally constant multi-sequence in $Q$.

Remark 3.3. The reader familiar with Descriptive Set Theory may suspect that this is not the most general definition. Simpson indeed considers Borel multi-sequences, namely multi-sequences whose range is countable and such that the preimage of any singleton is Borel. By the Galvin-Prikry Theorem [GP73], which is the Borel generalization of 2.11, any such multi-sequence admits a locally constant sub-multi-sequence. One can therefore safely replace "locally constant" by "Borel" in the above definition. While this result is very convenient in certain constructions and essential to some proofs, we shall not use it in this article.

Of course the definition of better-quasi-order can be formulated in terms of supersequences as Nash-Williams originally did. The only missing ingredient is a counterpart of the shift map $N \mapsto{ }_{*} N$ on finite subsets of natural numbers.

Definition 3.4. For $s, t \in[\omega]^{<\infty}$ we say that $t$ is a shift of $s$ and write $s \triangleleft t$ if there exists $X \in[\omega]^{\infty}$ such that

$$
s \sqsubset X \text { and } t \sqsubset{ }_{*} X \text {. }
$$

Definitions 3.5. Let $Q$ be a qo and $f: F \rightarrow Q$ be a super-sequence.

1. We say that $f$ is bad if whenever $s \triangleleft t$ in $F$, we have $f(s) \not \leq f(t)$.

2. We say that $f$ is good if there exists $s, t \in F$ with $s \triangleleft t$ and $f(s) \leq f(t)$.

Using the notations introduced in Section 2.4.

Lemma 3.6. Let $Q$ be a quasi-order.

1. If $h:[\omega]^{\infty} \rightarrow Q$ is locally constant and bad, then $h^{\downarrow}: F^{h} \rightarrow Q$ is a bad supersequence.

2. If $f: F \rightarrow Q$ is a bad super-sequence from a front on $X$, then $f^{\uparrow}:[X]^{\infty} \rightarrow Q$ is a bad locally constant multi-sequence.

Proof. 1. Suppose $h:[X]^{\infty} \rightarrow Q$ is locally constant and bad. Let us show that $h^{\downarrow}: F^{h} \rightarrow Q$ is bad. If $s, t \in F^{h}$ with $s \triangleleft t$, i.e. there exists $Y \in[X]^{\infty}$ such that $s \sqsubset Y$ and $t \sqsubset{ }_{*} Y$. Then $h^{\downarrow}(s)=h(Y)$ and $h^{\downarrow}(t)=h\left({ }_{*} Y\right)$ and since $h$ is assumed to be bad, we have $h^{\downarrow}(s) \not \leq h^{\downarrow}(t)$.

2. Suppose $f: F \rightarrow Q$ is bad from a front on $X$ and let $Y \in[X]^{\infty}$. There are unique $s, t \in F$ such that $s \sqsubset Y$ and $t \sqsubset{ }_{*} Y$, and clearly $f^{\uparrow}(Y)=f(s), f^{\uparrow}\left({ }_{*} Y\right)=f(t)$, and $s \triangleleft t$. Therefore $f^{\uparrow}(X) \not \leq f^{\uparrow}\left({ }_{*} X\right)$ holds. 
We finally have the equivalence between both definitions.

Corollary 3.7. A quasi-order $Q$ is a BQO if and only if there is no bad super-sequence in $Q$.

\subsection{First examples and finite stability}

Every constant super-sequence is good, so Theorem 2.12 can be reformulated:

Example 3.8. Every finite quasi-order is a better-quasi-order.

If $Q$ is a well-order and $h:[X]^{\infty} \rightarrow Q$ is a multi-sequence in $Q$, we can consider the sequence $\left(X_{n}\right)_{n \in \omega}$ in $[X]^{\infty}$ defined inductively by $X_{0}=X$ and $X_{n+1}={ }_{*} X_{n}$. As $Q$ is a well-order, $h\left(X_{n}\right) \mathbb{E}_{Q} h\left(X_{n+1}\right)$ implies $h\left(X_{n+1}\right)<_{Q} h\left(X_{n}\right)$. Since the sequence $\left(h\left(X_{n}\right)\right)_{n \in \omega}$ cannot be strictly decreasing in $Q$, there exists $n$ such that $h\left(X_{n}\right) \leq_{Q}$ $h\left(X_{n+1}\right)=h\left({ }_{*} X_{n}\right)$. We have obtained the following:

Example 3.9. Every well-order is a better-quasi-order.

Lemma 3.10. Suppose $h:\left(P, \leq_{P}\right) \rightarrow\left(Q, \leq_{Q}\right)$ is map such that $h(p) \leq h\left(p^{\prime}\right)$ implies $p \leq p^{\prime}$ for all $p, p^{\prime} \in P$. If $Q$ is $\mathrm{BQO}$, then $P$ is $\mathrm{BQO}$.

Proof. If $f: F \rightarrow P$ is a bad super-sequence in $P$, then $h \circ f: F \rightarrow Q$ is a bad super-sequence in $Q$.

Theorem 2.11 also gives the following easy closure property.

Proposition 3.11. If $\left(Q, \leq_{Q}\right)$ and $\left(P, \leq_{P}\right)$ are BQO, then so is $\left(P \cup Q, \leq_{P} \cup \leq_{Q}\right)$. In other words, a finite union of $\mathrm{BQO}$ is still $\mathrm{BQO}$.

Proof. Take a super-sequence $f: F \rightarrow P \cup Q$. Apply Theorem 2.11 to the partition $\left\{f^{-1}(P), f^{-1}(Q)\right\}$ of $F$ to get a sub-front $G$ of $F$ that defines a sub-super-sequence which ranges either in $P$ or in $Q$. Since both $P$ and $Q$ are BQO, $g$ is good, and in turn so is $f$.

The following dichotomy is an easy corollary of Theorem 2.11 which turns out to be quite useful when dealing with multi-sequences or super-sequences.

Proposition 3.12. Let $E$ be a set and $R$ a binary relation on $E$. Then every multisequence $f:[X]^{\infty} \rightarrow E$ admits a sub-multi-sequence $g:[Z]^{\infty} \rightarrow E$ such that either $g(Y) R g\left({ }_{*} Y\right)$ holds for all $Y \in[Z]^{\infty}$, or $g(Y) R g\left({ }_{*} Y\right)$ holds for no $Y \in[Z]^{\infty}$.

Proof. Define $c:[X]^{\infty} \rightarrow 2$ by $c(Y)=1$ if and only if $f(Y) R f\left({ }_{*} Y\right)$. Clearly $c$ is locally constant so by Corollary 3.7 there exists an infinite subset $Z$ of $X$ such that $c:[Z]^{\infty} \rightarrow 2$ is constant. The corresponding sub-multi-sequence $g=f \uparrow_{[Z]^{\infty}}:[Z]^{\infty} \rightarrow E$ is as desired.

The analogue result for super-sequences also holds and easily follows from the result for multi-sequences: 
Proposition 3.13. Let $E$ be a set and $R$ a binary relation on $E$. Then every supersequence $f: F \rightarrow E$ admits a sub-super-sequence $g: G \rightarrow E$ such that

either for all $s, t \in G, s \triangleleft t$ implies $g(s) R g(t)$,

or for all $s, t \in G, s \triangleleft t$ implies that $g(s) R g(t)$ does not hold.

When $\left(P, \leq_{P}\right)$ and $\left(Q, \leq_{Q}\right)$ are two quasi-orders, $\leq_{P} \times \leq_{Q}$ stands for the product quasi-order on $P \times Q$, that is

$$
(p, q) \leq_{P} \times \leq_{Q}\left(p^{\prime}, q^{\prime}\right) \longleftrightarrow p \leq_{p}^{\prime} \text { and } q \leq_{Q} q^{\prime} .
$$

Proposition 3.14. If $\left(Q, \leq_{Q}\right)$ and $\left(P, \leq_{P}\right)$ are $\mathrm{BQO}$, then so is $\left(P \times Q, \leq_{P} \times \leq_{Q}\right)$.

Therefore a finite product of $\mathrm{BQO} s$ is still $\mathrm{BQO}$.

Proof. Suppose that $f:[X]^{\infty} \rightarrow P \times Q$ is a bad multi-sequence and write $f(Y)=$ $\left(f_{P}(Y), f_{Q}(Y)\right)$. Then for every $Y \in[X]^{\infty}$ either $f_{P}(Y) \varliminf_{P} f_{P}\left({ }_{*} Y\right)$ or $f_{Q}(Y) \not_{Q}$ $f_{Q}\left({ }_{*} Y\right)$. Applying Proposition 3.12 to $f$ and the binary relation $(p, q) R\left(p^{\prime}, q^{\prime}\right)$ iff $p \leq_{P} p^{\prime}$ we obtain either that $f_{Q}$ is a bad multi-sequence in $\left(Q, \leq_{Q}\right)$ or that $f_{P}$ is a bad multi-sequence in $\left(P, \leq_{P}\right)$.

\subsection{The real deal: infinite stability}

We now turn to stability under infinitary operations, which was the original motivation behind the introduction of $\mathrm{BQO}$; to quote Marcone "the general pattern [is] that if a finitary operation preserves WQO then its infinitary version preserves BQO." More specifically, we are interested in operations $Q \mapsto O(Q)$ which are infinitary in the sense that each member of $O(Q)$ can be thought of as an infinite structure labeled by elements of $Q$. As an example we already have seen $Q \mapsto \mathcal{D}(Q)$. While Higman's Theorem ensures that finite sequences in a WQO again form a WQO, we now turn to the infinitary analogue: the operation of taking infinite sequences. Let us define what we mean by that, assuming basic knowledge concerning ordinals ${ }^{4}$.

Let $\left(Q, \leq_{Q}\right)$ be a qo. A transfinite sequence $\bar{q}$ in $Q$ is a map from an ordinal $\alpha$ to $Q$, $\alpha$ being then the length of $\bar{q}$, denoted by $|\bar{q}|$. The notation $Q^{\alpha}$ stands for the sequences in $Q$ of length $\alpha$, and $Q^{\mathrm{ON}}$ denotes the class of all transfinite sequences in $Q$. Given two sequences $\bar{q}$ and $\bar{p}$ in $Q^{\text {ON }}$ of respective lengths $\alpha$ and $\beta$, we write $\bar{q} \leq_{Q^{\text {ON }}} \bar{p}$ if there is an increasing injection $\iota: \alpha \rightarrow \beta$ satisfying $\bar{q}(\xi) \leq_{Q} \bar{p}(\iota(\xi))$ for all $\xi$ in $\alpha$. Notice in particular that $\bar{q} \leq_{Q^{\mathrm{ON}}} \bar{p}$ implies $|\bar{q}| \leq|\bar{p}|$.

Observe that for Rado's partial order $\mathfrak{R}$ from Example $1.2 \mathfrak{R}^{\omega}$ is not wQO, hence $Q \mapsto Q^{\mathrm{ON}}$ does not preserve wQOness. However $Q^{\mathrm{ON}}$ is BQO whenever $Q$ is BQO and we now outline the proof this result. The central element to this proof is the so-called Minimal Bad Lemma, that we state without proof. It is a key result in many theorems concerning BQO.

Definition 3.15. Let $\left(Q, \leq_{Q}\right)$ be a qo.

\footnotetext{
${ }^{4}$ As treated for instance in any introduction to set theory.
} 
- A partial ranking of $\left(Q, \leq_{Q}\right)$ is a well-founded quasi-order $\leq^{\prime}$ on $Q$ such that $p \leq^{\prime} q$ implies $p \leq_{Q} q$.

- Given any qo $\left(Q, \leq^{\prime}\right)$ and multi-sequences $f:[X]^{\infty} \rightarrow Q$ and $g:[Y]^{\infty} \rightarrow Q$ we write $f \leq^{\prime} g$ (resp. $f<^{\prime} g$ ) when we have both $X \subseteq Y$ and $f(Z) \leq^{\prime} g(Z)$ (resp. $\left.f(Z)<^{\prime} g(Z)\right)$ for all $Z \in[X]^{\infty}$.

- Given a partial ranking $\leq^{\prime}$ of $\left(Q, \leq_{Q}\right)$, a locally constant multi-sequence $g:[Y]^{\infty} \rightarrow$ $Q$ that is bad with respect to $\leq_{Q}$ is minimal bad if any locally constant multisequence $f$ satisfying $f<^{\prime} g$ is good.

Theorem 3.16 (Minimal Bad Lemma). Let $\leq^{\prime}$ be a partial ranking of a quasi-order $\left(Q, \leq_{Q}\right)$. If $f:[X]^{\infty} \rightarrow Q$ is a locally constant bad multi-sequence, then there is a locally constant multi-sequence $g \leq^{\prime} f$ that is minimal bad.

We refer the interested reader to [vEMS87] (see also [Mar95]) for a condensed proof of that result. Note however that we restricted ourselves here to talking about locally constant multi-sequences where in both of the above references the authors deal with Borel multi-sequences. This is not an issue since as we explained in Remark 3.3 every Borel multi-sequence admits a locally constant sub-multi-sequence.

We borrow the proof of the following lemma to [Sim85].

Lemma 3.17. Given $\left(Q, \leq_{Q}\right)$ a qo, if $\bar{q}$ and $\bar{p}$ in $Q^{\mathrm{ON}}$ satisfy $\bar{q} \varliminf_{Q^{\mathrm{ON}}} \bar{p}$ then there is $\theta<|\bar{q}|$ such that $\bar{q} \uparrow_{\theta} \leq_{Q^{\mathrm{ON}}} \bar{p}$ but $\bar{q} \uparrow_{\theta+1} \not_{Q^{\mathrm{ON}}} \bar{p}$.

Proof. Define a map $h:|\bar{q}| \rightarrow|\bar{p}|+1$ by induction: $h(\alpha)$ is the minimal $\xi<|\bar{p}|$ such that $\bar{q}(\alpha) \leq_{Q} \bar{p}(\xi)$ and $\xi>h(\beta)$ for all $\beta<\alpha$, if such a $\xi$ exists; and $h(\alpha)=|\bar{p}|$ otherwise. Notice that $\bar{q} \leq_{Q^{\text {ON }}} \bar{p}$ iff $h(\alpha)<|\bar{p}|$ for all $\alpha<|\bar{q}|$.

Now since $\bar{q} \not_{Q^{\text {ON }}} \bar{p}$, there is a minimal $\theta$ such that $h(\theta)=|\bar{p}|$. By minimality of $\theta$ we have $\bar{q} \uparrow_{\theta} \leq_{Q^{\mathrm{ON}}} \bar{p}$, and by definition of $h$ we have $\bar{q} \uparrow_{\theta+1} \not_{Q^{\mathrm{ON}}} \bar{p}$.

We are ready to prove stability under the taking of tranfinite sequences. As a matter of fact, we prove a stronger property that Louveau and Saint-Raymond in [LS90] call, in our terminolgy, reflection of bad multi-sequences.

Theorem 3.18 (Nash-Williams). If $\left(Q, \leq_{Q}\right)$ is a quasi-order and $f:[X]^{\infty} \rightarrow Q^{\mathrm{ON}}$ is a locally constant bad multi-sequence, then there is $Y \in[X]^{\infty}$ and a locally constant multi-sequence $\varphi$ from $[Y]^{\infty}$ to the ordinals such that $f \circ \varphi$ is a (locally constant) bad multi-sequence in $Q$.

Proof. We first define a partial ranking of $\left(Q^{\mathrm{ON}}, \leq_{Q^{\mathrm{ON}}}\right)$. For $\bar{q}$ and $\bar{p}$ in $Q^{\mathrm{ON}}$ define $\bar{q} \leq^{\prime} \bar{p}$ if and only if $\bar{q}$ is a prefix of $\bar{p}$, that is: there is $\theta \leq|\bar{p}|$ such that $\bar{q}=\left.\bar{p}\right|_{\theta}$. Clearly $\leq^{\prime}$ is a partial ranking.

Take a locally constant bad multi-sequence $f:[X]^{\infty} \rightarrow Q^{\mathrm{ON}}$, and apply Theorem 3.16 to get $X^{\prime} \in[X]^{\infty}$ and a locally constant minimal bad multi-sequence $g:\left[X^{\prime}\right]^{\infty} \rightarrow Q^{\text {ON }}$ such that $g \leq^{\prime} f$. For every $Z \in\left[X^{\prime}\right]^{\infty}$ let $\varphi(Z)$ be the unique ordinal $<|g(Z)|$ such that 
$\left.g(Z)\right|_{\varphi(Z)} \leq_{Q^{\mathrm{ON}}} g\left({ }_{*} Z\right)$ but $g(Z) \Gamma_{\varphi(Z)+1} \mathbb{\leq}_{Q^{\mathrm{ON}}} g\left({ }_{*} Z\right)$, the existence of which is granted by Lemma 3.17 .

Let us check that the map $\varphi:\left[X^{\prime}\right]^{\infty} \rightarrow$ ON is locally constant. For any $Z \in\left[X^{\prime}\right]^{\infty}$, since $g$ is locally constant there are basic open sets $U \ni Z$ and $V \ni{ }_{*} Z$ on which $g$ is constant. By continuity of the shift map, we can find a basic open set $W$ with $Z \in W \subseteq U$ such that ${ }_{*} Y \in V$ for all $Y \in W$. It follows that $\varphi$ is constant on $W$, as desired.

Notice that by definition of $\varphi(Z)$ we have $g(Z) \uparrow_{\varphi(Z)}<^{\prime} g(Z)$ for all $Z \in\left[X^{\prime}\right]^{\infty}$, so by minimality of $g$ the multi-sequence $Z \mapsto g(Z) \Gamma_{\varphi(Z)}$ is good. We can now apply Proposition 3.12 to obtain $Y \in\left[X^{\prime}\right]^{\infty}$ such that $g \uparrow_{[Y]^{\infty}}$ is perfect. So to sum up, we have for all $Z \in[Y]^{\infty}$ :

$$
g(Z) \Gamma_{\varphi(Z)} \leq_{Q^{\mathrm{ON}}} g\left({ }_{*} Z\right) \Gamma_{\varphi\left({ }_{*} Z\right)} \text { but } g(Z) \Gamma_{\varphi(Z)+1} \not_{Q^{\mathrm{ON}}} g\left({ }_{*} Z\right) \Gamma_{\varphi(* Z)+1} .
$$

This implies that for all $Z \in[Y]^{\infty}$ we have $g(Z)(\varphi(Z)) \not_{Q} g\left({ }_{*} Z\right)\left(\varphi\left({ }_{*} Z\right)\right)$. Notice finally that as $g(Z)$ is a prefix of $f(Z)$, we have $f \circ \varphi=g \circ \varphi$ which concludes the proof.

The previous result enjoys a converse, proven by Pouzet in [Pou72]:

Theorem 3.19 (Pouzet). A quasi-order $\left(Q, \leq_{Q}\right)$ is $\mathrm{BQO}$ if and only if $\left(Q^{\mathrm{ON}}, \leq_{Q^{\mathrm{ON}}}\right)$ is WQO.

This technique was instrumental in finding many examples of BQOs, the first and arguably most famous of these examples was found by Laver, a couple of years only after Nash-Williams' results. We recall it here, along with some others, in chronological order. We leave the interested reader look for precise definitions in the references.

Theorem 3.20. The following quasi-orders are $\mathrm{BQO}$ :

1. Embeddability between $\sigma$-scattered linear orders (Laver [Lav71]).

2. Surjective homomorphism between countable linear orders (Landraitis [Lan79]).

3. Embeddability between countable trees (Corominas [Cor85]).

4. Continuous embeddability between countable linear orders (van Engelen-MillerSteel [vEMS87]).

5. Embeddability between countable $N$-free partial orders (Thomassé [Tho00]).

We would like to point out that stronger versions of the above results are available, generally in the same articles as those cited. The general method for these proofs follows indeed the same pattern as Theorem 3.18, and what is proven is in general reflection of bad multi-sequences, which gives a BQO result for BQO-labelled structures. For more on this, see [LS90, Section 3].

That being said, there are other ways to prove that a certain class is BQO. The most famous involves games, it is due to Wadge and is called continuous reducibility. Once again, we mention some of them and leave the reader look for the specifics. 
Theorem 3.21. The following quasi-orders are $\mathrm{BQO}$ :

1. Continuous reducibility between Borel subsets of 0-dimensional Polish spaces (Wadge, Martin, van Engelen-Miller-Steel, see for instance [vEMS87]).

2. Embeddability between Borel sub-orders of $\mathbb{R}^{2}$ (Louveau-Saint Raymond, [LS90]).

3. Assuming Projective Determinacy, embeddability between Borel sub-orders of $\mathbb{R}^{n}$ for all $n \in \omega$ (Louveau-Saint Raymond, [LS90]).

4. Topological embeddability between 0-dimensional Polish spaces (see [Car13]).

In all these examples, BQO is used to prove that some quasi-order is WQO. We would now like to turn to some results of a somewhat different nature, focusing on yet another operation: passing from a quasi-order to the quasi-order of ideals. This is a central notion for the last two sections of this article.

Definition 3.22. Let $P$ be a partial order. An ideal of $P$ is a subset $I \subseteq P$ such that

1. $I$ is non empty;

2. $I$ is a downset;

3. for every $p, q \in I$ there exists $r \in I$ with $p \leq r$ and $q \leq r$.

We write $\operatorname{Id}(P)$ for the set of ideals of $P$ partially ordered by inclusion.

Equivalently, a subset $I$ of a po $P$ is an ideal if $I$ a downset and $I$ is directed, namely every (possibly empty) finite subset $F \subseteq I$ admits an upper bound in $I$, i.e. there exists $q \in I$ with $F \subseteq \downarrow q$. For every $p \in P$, the set $\downarrow p$ is an ideal called a principal ideal.

For each quasi-order $\left(Q, \leq_{Q}\right)$ we have the embedding

$$
\begin{aligned}
& Q \longmapsto \operatorname{Id}(Q) \\
& q \longmapsto \downarrow q,
\end{aligned}
$$

and we henceforth identify each element $p$ with the corresponding principal ideal $\downarrow p$. In particular we have the inclusions $P \subseteq \operatorname{Id}(P) \subseteq \mathcal{D}(P)$ as partial orders.

We observe that we cannot replace $\mathcal{D}(P)$ by $\operatorname{Id}(P)$ in Proposition 1.1, Item 3, i.e. it is not true that a po $P$ is WQO if and only $\operatorname{Id}(P)$ is well-founded. The simplest example is given by the antichain $A=(\omega,=)$. The partial order $\operatorname{Id}(A)$ is equal to $A$ so, in particular, even though $A$ is not WQO, $\operatorname{Id}(A)$ is well-founded. Nonetheless, when $P$ is WQO then $\operatorname{Id}(P)$ is well-founded.

A non-principal ideal of $P$ is an ideal which is not of the form $\downarrow p$ for some $p \in P$. We write $\operatorname{Id}^{*}(P)$ for the partial order of non-principal ideals, i.e. $\operatorname{Id}^{*}(P)=\operatorname{Id}(P) \backslash P$. The partial order $\operatorname{Id}^{*}(P)$ is therefore the remainder of the ideal completion of $P$.

The following result was conjectured by [Pou78] and proved by the authors in [CP14].

Theorem 3.23. Let $Q$ be WQO. If $\operatorname{Id}^{*}(Q)$ is BQO, then $Q$ is BQO. 
The first corollary of Theorem 3.23 that we mention is:

Corollary 3.24. If $Q$ is $\mathrm{WQO}$ and $\operatorname{Id}^{*}(Q)$ is finite, then $Q$ is $\mathrm{BQO}$.

This result is due to [Pou78] and a direct proof is presented by [Fra00, Chapter 7, 7.7.8].

This first simple corollary already allows us to prove the following proposition, a particular case of which was used by [Car13].

Proposition 3.25. Let $\varphi: \omega \rightarrow \omega$ be progressive, i.e. such that $n \leq \varphi(n)$ for every $n \in \omega$. Then the partial order $\leq_{\varphi}$ on $\omega$ defined by

$$
m \leq_{\varphi} n \quad \longleftrightarrow \quad m=n \text { or } \varphi(m)<n .
$$

is a better-quasi-order.

Proof. Let $g: \omega \rightarrow \omega$ be any sequence. Then either $g$ is bounded in the usual order and so $g$ is good for $\leq_{\varphi}$, or $g$ is unbounded in the usual order and so there exists $n$ such that $g(n)>\varphi(g(0))$ and so $g$ is good for $\leq_{\varphi}$. Hence $\left(\omega, \leq_{\varphi}\right)$ is WQO.

Now let $I$ be a non principal ideal in $\left(\omega, \leq_{\varphi}\right)$. In particular $I$ is an infinite subset of $\omega$, so for every $m \in \omega$ there exists $n \in I$ such that $\varphi(m)<n$ and so $m \leq_{\varphi} n \in I$. Therefore $I=\omega$ and so there is exactly one non-principal ideal of $\left(\omega, \leq_{\varphi}\right)$. It follows by Corollary 3.24 that $\left(\omega, \leq_{\varphi}\right)$ is BQO.

\section{In-between: an intractable diversity of inconspicuous orders}

The following classes of WQOs are sometimes considered as approximations of the concept of BQO.

Definition 4.1. Let $Q$ be a quasi-order and $1 \leq \alpha<\omega_{1}$. We say that $Q$ is $\alpha$-BQO if and only if every super-sequence $f: F \rightarrow Q$ with $\operatorname{rk} F \leq \alpha$ is good.

Remark 4.2. [Mar94], [PS06], for example, use a different definition of $\alpha$-BQO which is easily seen to be equivalent to ours.

Clearly a qo is WQO if and only if it is 1-BQO, and it is BQO if and only if its $\alpha$-BQO for every $\alpha<\omega_{1}$. Rado's poset, as shown in Example 1.2 is WQO but is not 2-BQO.

Marcone showed in [Mar94] that these notions are all distinct.

Theorem 4.3. For every countable ordinal $\alpha$ there exists a quasi-order that is $\beta$-BQO for all $\beta<\alpha$ but that is not $\alpha$-BQO.

One natural attempt to prove this theorem consists of considering on any front of rank equal to $\alpha$ the complement of the binary relation $\triangleleft$. This binary relation however fails to be a quasi-order" . Marcone's proof actually amounts to first showing that on "well

\footnotetext{
${ }^{5}$ The only notable exception is $[\omega]^{1}$ where both $\triangleleft$ and its complement are actually transitive. If $F$ is a front on $X=\left\{x_{0}, x_{1}, x_{2}, \ldots\right\}, Y=\left\{x_{3}, x_{4}, x_{5}, \ldots\right\}$ and if $s, t, u \in F$ are such that $s \sqsubset\left\{x_{0}\right\} \cup Y$, $|s| \geq 2, t \sqsubset\left\{x_{1}, x_{2}\right\} \cup Y,|t| \geq 2$ and $u \sqsubset Y$. Then $s \triangleleft u$, while neither $s \triangleleft t$ nor $t \triangleleft u$.
} 
chosen" fronts the complement of $\triangleleft$ lacks only transitivity in order to be the desired counter-example. Then one chooses an enumeration of the front at stake before using a result due to Pouzet to fix the transitivity (see [Mar94, Theorem 1.8]).

In this section we present examples of quasi-orders which are $n$-BQO but not $(n+1)$ BQO for each $n \geq 1$ and an example of a quasi-order which is not $\omega$-BQO but is $n$-BQO for all $n<\omega$. While their definition is simple and it is easy to see that they fail to be BQO, it does require some work to show they do enjoy a fair share of BQOness. Here we follow a new approach based on ideals and which relies on the following easy refinement of Theorem 3.23 whose proof can be found in the second author's PhD thesis [Peq15, Theorem 4.43]:

Theorem 4.4. For every $n \in \omega$, if $Q$ is $\mathrm{WQO}$ and $\operatorname{Id}^{*}(Q)$ is $n$-BQO then $Q$ is $(1+n)$-BQO.

Before going further, let us stop on our crucial example once again, to illustrate the rôle played by ideals.

Example 4.5 (Rado's poset continued). We continue on Rado's partial order $\Re$ defined in Example 1.2 and compute $\operatorname{Id}(\mathfrak{R})$. We claim that $\operatorname{Id}(\mathcal{R})=\mathcal{R} \cup\left\{I_{n} \mid n \in \omega\right\} \cup\{\top\}$ where $I_{n}=\downarrow\{\{n, k\} \mid n<k\}$ for $n \in \omega$ and $T=\mathcal{R}$. We have $\{m, n\} \leq I_{k}$ if and only if $m=k$ or $n<k$, and $a \leq \top$ for all $a \in \operatorname{Id}(\mathcal{R})$. The non principal ideals are the $I_{n} \mathrm{~s}$ and $T$. We show there are no other ideals. Let $I$ be an ideal of $\mathcal{R}$. First suppose for all $k \in \omega$ there exists $\{m, n\} \in I$ with $k<m$, then $I=\top$. Suppose now that there exists $m=\max \{k \mid \exists l\{k, l\} \in I\}$. If there is infinitely many $n$ such that $\{m, n\} \in I$ then $I=I_{m}$. Otherwise $I=\downarrow\{m, n\}$ for $n=\max \{l \mid\{m, l\} \in I\}$.

Observe that $\left(I_{n}\right)_{n \in \omega}$ is an antichain in $\operatorname{Id}(\mathcal{R})$, hence a bad sequence witness to the fact that $\operatorname{Id}(\mathcal{R})$ is not WQO.

Here is the definition of the explicit counter-examples that we know. The quasi-orders defined on fronts of finite ranks first appeared in [Pou72] and the quasi-order on $\mathcal{S}$ was defined by Assous-Pouzet [PA].

Definition 4.6. For every $n \geq 1$ we let $\mathfrak{R}_{n}=\left([\omega]^{n}, R_{n}\right)$ where $R_{n}$ is the binary relation defined by

$$
s R_{n} t \longleftrightarrow\left\{\begin{array}{l}
s(i) \leq t(i), \text { for all } i<n, \text { and } \\
\text { if } s(0)<t(0), \text { then there is } j>0 \text { with } s(j)<t(j-1) .
\end{array}\right.
$$

Furthermore, we let $\mathfrak{R}_{\omega}=\left(\mathcal{S}, R_{\omega}\right)$ where $\mathcal{S}=\left\{s \in[\omega]^{<\infty}:|s|=s(0)+1\right\}$ is the Schreier barrier and:

$$
s R_{\omega} t \longleftrightarrow\left\{\begin{array}{l}
\text { if } s(0)=t(0), \text { then } s(i) \leq t(i) \text { for all } i<|s|, \text { and } \\
\text { if } s(0)<t(0), \text { then there is } n \leq|s| \text { such that } s \uparrow_{n} R_{n} t \uparrow_{n} .
\end{array}\right.
$$

Notice that $\mathfrak{R}_{1}$ is simply $\left([\omega]^{1},=\right)$ and that $\mathfrak{R}_{2}$ is exactly Rado's poset $\mathfrak{R}$ from Example 1.2. Moreover it easy to check that the binary relations we defined are included in the complement of the binary relation $\triangleleft$. 
Lemma 4.7. Each of the $\mathfrak{R}_{n}$ for $n \geq 2$ as well as $\mathfrak{R}_{\omega}$ are WQOs.

Proof. One needs to check that $R_{\omega}$ and each $R_{n}$ is transitive. We only treat one specific case which is a main obstacle to generalizing this idea to arbitrary fronts. Let $s, t, u \in \mathcal{S}$ with $s R_{\omega} t$ and $t R_{\omega} u$. Clearly $|s| \leq|u|$ and $s(i) \leq u(i)$ for all $i<|s|$. Now suppose that $s(0)=t(0)$ and $t(0)<u(0)$, so $s(0)<u(0)$. Then there exists $n \leq|t|$ such that $t R_{n} u$. By definition of $\mathcal{S}, s(0)=t(0)$ implies $|s|=|t|$, so we also have $n \leq|s|$. Since $s \uparrow_{n} R_{n} t \uparrow_{n}$ by transitivity of $R_{n}$ we get $s \uparrow_{n} R_{n} u \uparrow_{n}$ and so $s R_{\omega} u$ in this case, as desired.

Next we show that $\mathfrak{R}_{\omega}$ is WQO. The case of $\mathfrak{R}_{n}$ for $n \geq 2$ is similar. Suppose towards a contradiction that $\left(s_{n}\right)_{n \in \omega}$ is a bad sequence in $\mathfrak{R}_{\omega}$ and let $m_{n}=\min s_{n}$ for each $n$. By possibly going to a subsequence, we can assume that either $\left(m_{n}\right)_{n}$ is unbounded or else $\left(m_{n}\right)_{n}$ is constant equal to some $k$. In the latter case, it follows that the sequence $t_{n}=s_{n} \backslash\left\{m_{n}\right\}, n \in \omega$, is a bad sequence in $\omega^{k}$ with the pointwise ordering, a contradiction. In the former case, we can find $i$ with $m_{i}=\left|s_{i}\right| \geq 2$ and then $j>i$ such that $m_{j}>\max s_{i}$, so that we get $s_{i} R_{m_{i}} s_{j}\left\lceil m_{i}\right.$. This implies that $s_{i} R_{\omega} s_{j}$, a contradiction again.

While it is very tempting to try to generalize the above definition to arbitrary fronts, one should notice that very specific properties of the fronts $[\omega]^{n}$ and $\mathcal{S}$ are needed to prove their transitivity. As a matter of fact, so far any attempt to generalize these examples has failed to be transitive.

We now compute the ideals of $\Re_{n+1}$.

Definition 4.8. For every $n \geq 1$, let we define $\mathfrak{I}_{n}=\left([\omega] \leq n, S_{n}\right)$ where

$$
s S_{n} t \longleftrightarrow\left\{\begin{array}{l}
|s| \geq|t| \text { and } s(i) \leq t(i) \text { for all } i<|t|, \text { and } \\
\text { if }|s|=|t|=n, \text { then } s R_{n} t .
\end{array}\right.
$$

where $R_{n}$ is the relation from Definition 4.6.

Proposition 4.9. For ever natural number $n \geq 1, \operatorname{Id}^{*}\left(\mathfrak{R}_{n+1}\right)$ is isomorphic to $\mathfrak{I}_{n}$.

Proof. For $s \in[\omega]^{\leq n}$ we say that a sequence $\left(s_{i}\right)_{i \in \omega}$ in $\mathfrak{R}_{n+1}$ is good for $s$ if

1. $s \sqsubset s_{i}$ for all $i$,

2. $\left(s_{i}(|s|)\right)_{i \in \omega}$ is strictly increasing, and

3. $\left(s_{i}\right)_{i \in \omega}$ is strictly increasing in $\mathfrak{R}_{n+1}$.

Let $s, t \in[\omega]^{\leq n}$ and suppose that $\left(s_{i}\right)_{i \in \omega}$ and $\left(t_{i}\right)_{i \in \omega}$ are good for $s$ and $t$, respectively. We claim that if $\downarrow\left\{s_{i} \mid i \in \omega\right\} \subseteq \downarrow\left\{t_{i} \mid i \in \omega\right\}$ (where the downward closure is taken in $\left.\mathfrak{R}_{n+1}\right)$ then $s S_{n} t$. So in particular if $\downarrow\left\{s_{i} \mid i \in \omega\right\}=\downarrow\left\{t_{i} \mid i \in \omega\right\}$, then $s=t$.

First suppose towards a contradiction that $|s|<|t|$. There exists $k$ such that $\left.s_{k}(|s|)\right\rangle$ $t(|s|)$ and so $s_{k} \notin \downarrow\left\{t_{i} \mid i \in \omega\right\}$ since for no $j$ we have $s_{k} R_{n+1} t_{j}$. A contradiction, hence $|s| \geq|t|$. Since there exists $i$ such that $s_{0} R_{n+1} t_{i}$, it follows in particular that $s(j) \leq t(j)$ for all $j<|t|$. Finally assume that $|s|=|t|=n$ and that $s(0)<t(0)$. There is $i$ such 
that $s_{i}(|s|) \geq t(|s|-1)$ and there is $j$ such that $s_{i} R_{n+1} t_{j}$. By definition of $R_{n+1}$ there is $k>0$ such that $s_{i}(k)<t_{j}(k-1)$. Since necessarily $k<|s|$, it follows that $k$ is also a witness to the fact that $s R_{n} t$. This proves the claim.

Next we see that for every $s \in[\omega]^{\leq n}$ there exists a sequence $\left(s_{i}\right)_{i \in \omega}$ which is good for $s$. One easily checks that $s_{i}=\{2 i, 2 i+1, \ldots, 2 i+n\}, i \in \omega$, is good for $\emptyset$. Moreover if $s \in[\omega]^{\leq n}$ is not empty and we let $s_{i} \in[\omega]^{n+1}$ with $s_{i} \sqsubset s \cup \omega /(i+\max (s))$ for each $i \in \omega$, then $\left(s_{i}\right)_{i \in \omega}$ is good for $s$.

For every $s \in[\omega]^{\leq n}$, let $I_{s}=\downarrow\left\{s_{i} \mid i \in \omega\right\}$ for some sequence $\left(s_{i}\right)_{i \in \omega}$ that is good for $s$. By the claim this is a well defined map $\mathfrak{I}_{n} \rightarrow \operatorname{Id}^{*}\left(\mathfrak{R}_{n+1}\right)$ and moreover $I_{s} \subseteq I_{t}$ implies $s S_{n} t$.

To see this map is surjective, let $I$ be a non principal ideal of $\mathfrak{R}_{n+1}$. Since $I$ is a (countable) non principal ideal, there exists a strictly $R_{n+1}$-increasing sequence $\left(s_{i}\right)_{i \in \omega}$ such that $I=\downarrow\left\{s_{i} \mid i \in \omega\right\}$. By repeated application of the Ramsey Theorem and possibly going to a subsequence, we can assume that for all $j<n+1$ the sequence $\left(s_{i}(j)\right)_{i \in \omega}$ is either constant or strictly increasing. Since $\left(s_{i}\right)_{i \in \omega}$ has no constant subsequence, this implies that there exists $s_{I} \in[\omega]^{\leq n}$ such that $\left(s_{i}\right)_{i \in \omega}$ is good for $s_{I}$, as desired.

Finally we prove that $s S_{n} t$ implies $I_{s} \subseteq I_{t}$. Let $\left(s_{i}\right)_{i \in \omega}$ and $\left(t_{i}\right)_{i \in \omega}$ be good for $s$ and $t$ respectively. We distinguish two cases. First assume that $|t|<n$, then for every $i$ there exists $j$ with $s_{i}(n)<t_{j}(|t|)$, which together with $s S_{n} t$ implies $s_{i} R_{n+1} t_{j}$ and so $I_{s} \subseteq I_{t}$. Next assume that $|s|=|t|=n$ and so $s R_{n} t$. Then for all $i$ there is $j$ with $s_{i}(n) \leq t_{j}(n)$, and this implies that $s_{i} R_{n+1} t_{j}$.

We can now prove that each $\mathfrak{R}_{n}$ is the counter-example we wanted.

Theorem 4.10 (Pouzet). For all $n \geq 1, \mathfrak{R}_{n+1}$ is $n$-BQO but not $(n+1)$-BQO.

Proof. As we already observed, the identity map $[\omega]^{n+1} \rightarrow[\omega]^{n+1}$ is a bad super-sequence of rank $n+1$ in $\Re_{n+1}$, so it is not $(n+1)$-BQO. We prove that $\Re_{n+1}$ is $n$-BQO by induction on $n$. For $n=1$, we already know that $\mathfrak{R}_{2}=\mathfrak{R}$ is WQO. So let $n>1$ and assume that $\mathfrak{R}_{n}$ is $(n-1)$-BQO. We showed in Lemma 4.7 that $\mathfrak{R}_{n+1}$ is WQO and by Proposition 4.9 $\operatorname{Id}^{*}\left(\mathfrak{R}_{n+1}\right)$ is isomorphic to $\mathfrak{I}_{n}$. We show that $\mathfrak{I}_{n}$ is $(n-1)$-BQO and we then conclude the proof by Theorem 4.4 .

Notice that if $P_{k}=\left([\omega]^{k}, \leq^{k}\right)$ denotes the pointwise ordering where $s \leq^{k} t$ iff $s(i) \leq t(i)$ for all $i<k$, then $P_{k}$ is easily seen to be BQO by Lemma 3.10 and Proposition 3.14. Moreover by induction hypothesis $\Re_{n}$ is $(n-1)$-BQO, and therefore as in Proposition 3.11 it follows that $\Re_{n} \cup \bigcup_{k=0}^{n-1} P_{k}$ is also $(n-1)$-BQO. One easily checks that the identity map $\mathfrak{I}_{n} \rightarrow \mathfrak{R}_{n} \cup \bigcup_{k=0}^{n-1} P_{k}$ allows to conclude as in Lemma 3.10 that $\mathfrak{I}_{n}$ is $(n-1)$-BQO, as desired.

Using the above result we can prove that $\mathfrak{R}_{\omega}$ is $n$-BQO for all $n$.

Theorem 4.11 (Pouzet-Assous). The qo $\mathfrak{R}_{\omega}$ is $n$-BQO for all $n \in \omega$, but it is not $\omega$-BQO.

Proof. As before, the identity map is a bad super-sequence of rank $\omega$ in $\mathfrak{R}_{\omega}$, so it remains to show that $\mathfrak{R}_{\omega}$ is $n$-BQO for all $n$. Take $F$ a front of rank $n, f: F \rightarrow \mathcal{S}$ a super-sequence in $\mathfrak{R}_{\omega}$, and let us prove that it is good. Using the fact that $\omega$ is BQO and 
applying Proposition 3.12, we can assume by possibly going to a sub-super-sequence that $s \triangleleft t$ in $F$ implies $f(s)(0) \leq f(t)(0)$. Applying Proposition 3.12 again, we can further assume that either $s \triangleleft t$ in $F$ implies $f(s)(0)=f(t)(0)$, or $s \triangleleft t$ in $F$ implies $f(s)(0)<f(t)(0)$.

In the former case, there actually exists $i$ such that $f(s)(0)=i$ for all $s \in F$. This is because the transitive closure $\triangleleft^{*}$ of $\triangleleft$ inside $F$ is directed; notice indeed that for any $s$ and $t$ in $F$, if $u \in F$ satisfies $\max (s \cup t)<\min (u)$ then both $s \triangleleft^{*} u$ and $t \triangleleft^{*} u$ hold. But for each $i$ the restriction of $R_{\omega}$ to $\{s \in \mathcal{S} \mid s(0)=i\}$ is BQO since $\omega$ is BQO and any finite products of BQO is BQO by Proposition 3.14.

We now suppose that $s \triangleleft t$ in $F$ implies $f(s)(0)<f(t)(0)$. Applying Theorem 2.11 to the subset of $F$ given by $\{s \in F \mid f(s)(0)<n\}$ where $n$ is the rank of $F$, we obtain a sub-super-sequence which falls into one of the two following cases.

Either $f(s)(0)<n$ for every $s \in F$ and so $f$ is good since for each $i<n$ the restriction of $R_{\omega}$ to $\{s \in \mathcal{S} \mid s(0)=i\}$ is BQO and a finite union of BQOs is BQO by Proposition 3.11.

Or $f(s)(0) \geq n$ for every $s \in F$, and so $|f(s)| \geq n+1$ by definition of the Schreier barrier $\mathcal{S}$. In this case, we consider the super-sequence $g: F \rightarrow \mathfrak{R}_{n+1}$ given by $g(s)=$ $\left.f(s)\right|_{n+1}$. Since $\mathfrak{R}_{n+1}$ is $n$-BQO by Theorem 4.10, it follows that $g$ is good and there exists $s, t \in F$ with $s \triangleleft t$ and $g(s) R_{n+1} g(t)$. As $f(s)(0)<f(t)(0)$ and $\left.f(s)\right|_{n+1} R_{n+1}$ $f(t)\left\lceil_{n+1}\right.$, we have $f(s) R_{\omega} f(t)$ and therefore $f$ is good as desired.

\section{Classes of bqos definable by forbidden pattern}

We have seen in the last Section that a great variety of quasi-orders distinguish the notion of WQO from that of BQO. Importantly, these two concepts also are of distinct descriptive complexity: while the set of WQOs on $\omega$ is a $\Pi_{1}^{1}$-complete subset of the Cantor space, that of BQOs is $\boldsymbol{\Pi}_{2}^{1}$-complete as proved by Marcone [Mar95, Mar94]. In particular, while $Q$ is WQO iff neither $(\omega,=)$ nor $\omega^{\text {op }}$ (the opposite of $\omega$ ) embeds into $Q$, BQO cannot be defined by forbidding pattern, at least not in any relevant way. ${ }^{6}$

However in some particular cases, forbidding finitely many patterns do imply BQOness. In this section, we deduce results of this kind from Theorem 3.23.

\subsection{Interval orders}

Suppose that $Q$ is a WQO such that $\operatorname{Id}^{*}(Q)$ is a well-order. Since by Example 3.9 wellorders are BQO, such quasi-orders are BQO by Theorem 3.23.

Observe that when $Q$ is WQO, since ideals are downsets and $\mathcal{D}(Q)$ is well-founded, $\operatorname{Id}^{*}(Q)$ is well-founded too. Hence, if $Q$ is wQO then $\operatorname{Id}^{*}(Q)$ is linearly ordered if and only if $\operatorname{Id}^{*}(Q)$ is a well-order.

\footnotetext{
${ }^{6}$ Suppose that $\mathcal{B}$ is a basis for the set of BQOs on $\omega$, i.e. $B \subseteq 2^{\omega \times \omega}$ and for every qo $Q$ on $\omega$ we have $Q$ is BQO iff for no $B \in \mathcal{B}$ there exists an embedding from $B$ to $Q$. Then $\mathcal{B}$ is not analytic. Otherwise

$$
Q \text { is } \mathrm{BQO} \longleftrightarrow \text { there exists no } B \in \mathcal{B} \text { such that } B \text { embeds in } Q
$$

is a co-analytic definition of the set of BQOs on $\omega$, a contradiction with Marcone's Theorem.
} 
What are the quasi-orders whose non principal ideals are linearly ordered? Well, assume $Q$ is a quasi-order and that $I, J \in \operatorname{Id}^{*}(Q)$ are incomparable for inclusion. Let $p \in I \backslash J$ and $q \in J \backslash I$. Then $p$ is incomparable with $q$. Forbidding antichains of size 2 in $Q$ is simply asking that $Q$ is a linear order, and of course well-orders are BQO. But we can do better: since $I$ and $J$ are non principal, there are $p^{\prime} \in I$ with $p<p^{\prime}$ and $q^{\prime} \in J$ with $q<q^{\prime}$. The restriction of the quasi-order on $Q$ to $\left\{p, q, p^{\prime}, q^{\prime}\right\}$ is isomorphic to the partial order:

$$
2 \oplus 2=\lceil
$$

and therefore $2 \oplus 2$ embeds into $Q$. We are naturally led to following definition which appears frequently in the literature.

Definition 5.1. A partial order $P$ is an interval order if the partial order $2 \oplus 2$ does not embed into $P$. In other words for every $p, q, x, y \in P, p<x$ and $q<y$ imply $p<y$ or $q<x$.

The preceding discussion yields the following which is already stated in [PS06].

Theorem 5.2. An interval order is BQO if and only if it is WQO.

Notice that this theorem can be rephrased as follows: a partial order $P$ such that neither $(\omega,=)$, nor $\omega^{\text {op }}$, nor $2 \oplus 2$ embeds into $P$ is a better-quasi-order.

It appears that the notion of interval order was first studied by the twenty-yearsold Norbert Wiener [Wie14] who credits Bertrand Russell for suggesting the subject [FM92]. Wiener was later acknowledged as the originator of cybernetics [CS06]. The Reverse Mathematics of interval orders is studied in [Mar07].

For $p \in P$, let $\operatorname{Pred}(p)=\{q \in P \mid q<p\}$. It is easy to see that a partial order $P$ is an interval order if and only if the set $\{\operatorname{Pred}(p) \mid p \in P\}$ is linearly ordered by inclusion.

The terminology "interval order" was introduced by [Fis70] and stems from the following characterisation.

A non trivial closed interval of a partial order $Q$ is a set of the form $[a, b]=\{q \in Q \mid$ $a \leq q \leq b\}$ for some $a, b \in Q$ with $a<b$. We partially order the set $\operatorname{Int}(Q)$ of non trivial closed intervals of $Q$ by $[a, b] \leq[c, d]$ if and only if $a=c$ and $b=d$ or $b \leq c$.

For a partial order $P$ let us say that a map $I: P \rightarrow \operatorname{Int}(Q)$ is an interval representation of $P$ in $Q$ if for every $x, y \in P$ we have $x<y \leftrightarrow I(x)<I(y)$.

Let us first see that any partial order $P$ admits an interval representation. Let $\operatorname{Pred}^{+}(p)=\bigcap_{p<x} \operatorname{Pred}(x)$ and

$$
Q_{P}=\{\operatorname{Pred}(p) \mid p \in P\} \cup\left\{\operatorname{Pred}^{+}(p) \mid p \in P\right\}
$$

be partially ordered by inclusion.

Proposition 5.3 ([Bog93]). Let $P$ be a partial order. The map

$$
\begin{aligned}
I: P & \longrightarrow \operatorname{Int}\left(Q_{P}\right) \\
& \longmapsto \\
& \longmapsto I_{p}=\left(\operatorname{Pred}(p), \operatorname{Pred}^{+}(p)\right)
\end{aligned}
$$

is an interval representation of $P$ in $Q_{P}$. 
Proof. First observe that for every $p \in P$ we have $\operatorname{Pred}(q) \subset \operatorname{Pred}^{+}(q)$ since $q<p$ imply $q \in \operatorname{Pred}(x)$ for all $x>p$, and in fact $p \in \operatorname{Pred}^{+}(p) \backslash \operatorname{Pred}(p)$. So $I$ is well defined. If $p<q$, then $\operatorname{Pred}^{+}(p)=\bigcap_{p<x} \operatorname{Pred}(x) \subseteq \operatorname{Pred}(q)$, and so $I_{p}<I_{q}$. Conversely if $I_{p}<I_{q}$, then $\operatorname{Pred}^{+}(p) \subseteq \operatorname{Pred}(q)$ and since $p \in \operatorname{Pred}^{+}(p)$ we have $p<q$. Hence $I$ is an interval representation of $P$.

The following is a slight generalisation of a theorem by [Fis70]. The proof we give here is due to [Bog93].

Proposition 5.4. A partial order $P$ is an interval order if and only if there exists an interval representation of $P$ in some linear order.

Proof. Suppose $I: P \rightarrow \operatorname{Int}(L)$ is an interval representation of $P$ in a linear order $L$ and let $p_{0}<p_{1}$ and $q_{0}<q_{1}$ in $P$. If $I\left(p_{i}\right)=\left[l_{i}, r_{i}\right]$ and $I\left(q_{i}\right)=\left[m_{i}, s_{i}\right]$ then $r_{0} \leq l_{1}$ and $s_{0} \leq m_{1}$. Since $L$ is linearly ordered, either $r_{0} \leq m_{1}$ and so $p_{0}<q_{1}$, or $m_{1} \leq r_{0}$ and so $q_{0}<p_{1}$. Therefore $P$ is an interval order.

Conversely, suppose $P$ is an interval order. By Proposition 5.3, it suffices to prove the $Q_{P}$ is linearly ordered. But $\{\operatorname{Pred}(p) \mid p \in P\}$ is linearly ordered and $\operatorname{Pred}^{+}(p)=$ $\bigcap_{p<x} \operatorname{Pred}(x)$ is incomparable for the inclusion with some $X \in Q_{P}$ if and only if $\operatorname{Pred}(x)$ is incomparable with $X$ for some $x>p$.

\subsection{More classes of better-quasi-orders via forbidden patterns}

In fact continuing the above discussion we find that for any qo $Q, \operatorname{Id}^{*}(Q)$ is linearly ordered if and only if the po

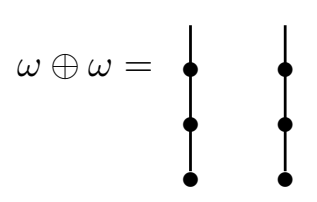

does not embed into $Q$. We therefore have the following:

Theorem 5.5. If neither $(\omega,=)$, nor $\omega^{\mathrm{op}}$, nor $\omega \oplus \omega$ embed into $Q$, then $Q$ is BQO.

Suppose now for a partial order $P$ that there exists a natural number $n$ such that the size of every antichain of $P$ is bounded by $n$. Then, by a theorem due to [Dil50], for $A$ an antichain of maximum size, say $n$, there exist subsets $P_{i}, i \in n$, such that $\left|P_{i} \cap A\right|=1$, $P_{i}$ is linearly ordered and $\bigcup_{i \in n} P_{i}=P$ (see also [Fra00, 4.14.1, p. 141]). In particular, if $P$ is further assumed to be well-founded, then $P$ is BQO as a finite union of well-orders.

Continuing further the discussion of the previous subsection, we see that if there exists an antichain $A$ of size $n$ among the non principal ideals of a qo $Q$, then the partial order 


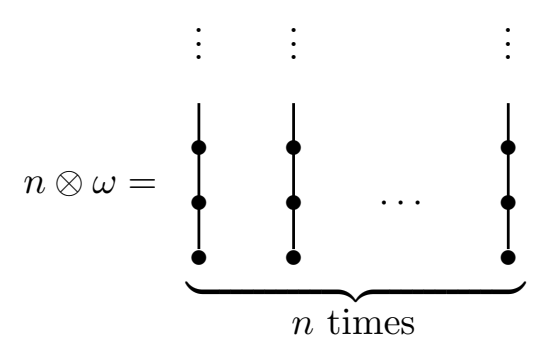

embeds into $Q$. Indeed, assume that $\left\{I_{i} \mid i \in n\right\}, n \geq 2$ is an antichain of non principal ideals of a qo $Q$. For each $i \in n$ and every $j \in n$ with $i \neq j$, since $I_{i} \nsubseteq I_{j}$ we can pick $q_{j} \in I_{i} \backslash I_{j}$ and by the fact that $I_{i}$ is directed there is $q^{i} \in I_{i}$ with $q^{i} \notin I_{j}$ for every $j \neq i$. Now since each $I_{i}$ is non principal there exists a strictly increasing sequence $\left(q_{k}^{i}\right)_{k \in \omega}$ in $I_{i}$ with $q_{0}^{i}=q^{i}$. This clearly yields and embedding of $n \otimes \omega$ into $\bigcup_{i \in n} I_{i}$. Therefore

Theorem 5.6. Let $n \geq 1$. If neither $(\omega,=)$, nor $\omega^{\mathrm{op}}$, nor $n \otimes \omega$ embed into $Q$, then $Q$ is BQO.

In this theorem, for each $n \geq 1$, we have a class of BQO which is defined by finitely many forbidden patterns. Examples of classes of BQOs defined by mean of forbidden patterns - left alone by finitely many - are quite rare. In fact to our knowledge the previous theorem is the best result of this sort.

\section{References}

[Bog93] Kenneth P Bogart. An obvious proof of Fishburn's interval order theorem. Discrete Mathematics, 118(1):239-242, 1993.

[Car13] Raphaël Carroy. A quasi-order on continuous functions. Journal of Symbolic Logic, 78(2):633-648, 2013.

[Cor85] Ernesto Corominas. On better quasi-ordering countable trees. Discrete Mathematics, 53:35-53, 1985.

[CP14] Raphaël Carroy and Yann Pequignot. From well to better, the space of ideals. Fundamenta Mathematicae, 227(3):247-270, 2014.

[CS06] Florence D. Conway and Jim Siegelman. Dark Hero of the Information Age: In Search of Norbert Wiener - The Father of Cybernetics. Basic Books, 2006.

[Di150] Robert P Dilworth. A decomposition theorem for partially ordered sets. Annals of Mathematics, pages 161-166, 1950.

[Fis70] Peter C Fishburn. Intransitive indifference with unequal indifference intervals. Journal of Mathematical Psychology, 7(1):144-149, 1970. 
[FM92] Peter Fishburn and Bernard Monjardet. Norbert Wiener on the theory of measurement 19141915 1921. Journal of Mathematical Psychology, 36(2):165-184, 1992.

[Fra48] Roland Fraïssé. Sur la comparaison des types d'ordres. C. R. Acad. Sci. Paris, 226:1330-1331, 1948.

[Fra00] Roland Fraïssé. Theory of Relations. Studies in Logic and the Foundations of Mathematics. Elsevier Science, 2000.

[GP73] Fred Galvin and Karel Prikry. Borel sets and Ramsey's theorem. The Journal of Symbolic Logic, 38:193-198, 61973.

[Hig52] Graham Higman. Ordering by divisibility in abstract algebras. Proceedings of the London Mathematical Society, 3(1):326-336, 1952.

[Kru72] Joseph Bernard Kruskal. The theory of well-quasi-ordering: A frequently discovered concept. Journal of Combinatorial Theory, Series A, 13(3):297305, 1972.

[Lan79] Charles Landraitis. A combinatorial property of the homomorphism relation between countable order types. Journal of Symbolic Logic, 44:403-411, 1979.

[Lav71] Richard Laver. On Fraïssé's order type conjecture. The Annals of Mathematics, 93(1):89-111, 1971.

[Lav76] Richard Laver. Well-quasi-orderings and sets of finite sequences. Mathematical Proceedings of the Cambridge Philosophical Society, 79(01):1-10, 1976.

[LS90] Alain Louveau and Jean Saint Raymond. On the quasi-ordering of Borel linear orders under embeddability. Journal of Symbolic Logic, 55(2):537-560, 1990.

[Mar94] Alberto Marcone. Foundations of bqo theory. Transactions of the American Mathematical Society, 345(2):641-660, 1994.

[Mar95] Alberto Marcone. The set of better quasi orderings is $\pi_{2}^{1}$-complete. Mathematical Logic Quarterly, 41:373-383, 1995.

[Mar05] Alberto Marcone. Wqo and bqo theory in subsystems of second order arithmetic. In Stephen G. Simpson, editor, Reverse Mathematics 2001, volume 21. Association for Symbolic Logic, 2005.

[Mar07] Alberto Marcone. Interval orders and reverse mathematics. Notre Dame J. Formal Logic, 48(3):425-448, 072007.

[NW65] Crispin St. John Alvah Nash-Williams. On well-quasi-ordering transfinite sequences. In Proc. Cambridge Philos. Soc, volume 61, pages 33-39. Cambridge University Press, 1965. 
[PA] Maurice Pouzet and Roland Assous. Personal communication.

[Peq15] Yann Pequignot. Better-quasi-order: ideals and spaces. Phd thesis, Université de Lausanne and Université Paris Diderot - Paris 7, 2015.

[Pou72] Maurice Pouzet. Sur les prémeilleurordres. Ann. Inst. Fourier (Grenoble), 22:1-20, 1972.

[Pou78] Maurice Pouzet. Sur la théorie des relations. Thèse d'état, Université Claude Bernard, 1978.

[Pou85] Maurice Pouzet. Applications of well quasi-ordering and better quasiordering. In Graphs and order, pages 503-519. Springer, 1985.

[PR82] Pavel Pudlák and Vojtěch Rödl. Partition theorems for systems of finite subsets of integers. Discrete Mathematics, 39(1):67-73, 1982.

[PS06] Maurice Pouzet and Norbert Sauer. From well-quasi-ordered sets to betterquasi-ordered sets. Journal of Combinatorics, 13(4):101, 2006.

[Rad54] Richard Rado. Partial well-ordering of sets of vectors. Mathematika, 1(02):8995, 1954 .

[RS04] Neil Robertson and P.D. Seymour. Graph minors. xx. wagner's conjecture. Journal of Combinatorial Theory, Series B, 92(2):325 - 357, 2004. Special Issue Dedicated to Professor W.T. Tutte.

[Sim85] Stephen G. Simpson. Bqo theory and Fraïssé's conjecture. In Richard Mansfield and Galen Weitkamp, editors, Recursive aspects of descriptive set theory, pages 124-138. Oxford University Press, 1985.

[Tho00] Stephan Thomassé. On better-quasi-ordering countable series-parallel orders. Transactions of the American Mathematical Society, 352(6):2491-2505, 2000.

[vEMS87] Fons van Engelen, Arnold W Miller, and John Steel. Rigid Borel sets and better quasiorder theory. Contemporary mathematics, 65, 1987.

[Wie14] Norbert Wiener. A contribution to the theory of relative position. In Proc. Camb. Philos. Soc, volume 17, pages 441-449, 1914. 